\title{
"Una donna con la D maiuscola". Analisi di Bésame Giuda di Carmen Consoli
}

\author{
Veronica Ricotta
}

PUBBLICATO: 31 DICEMBRE 2019

\section{Cantantessa}

P assando dalle cantanti interpreti (Mina, Vanoni) alle cantautrici abbiamo scelto di chiudere il nostro percorso con Carmen Consoli, estrapolando dal corpus esaustivo delle canzoni della cantante un testo di cui qui si presenta un'analisi di tipo linguistico volta a isolare alcuni elementi, con particolare attenzione alla selezione lessicale, e alla rappresentazione dello stereotipo di genere. I testi delle canzoni della Consoli hanno suscitato l'interesse dei linguisti, per esempio per la metrica, come nei lavori di Giuseppe Antonelli, e per l'uso del dialetto, come nelle analisi di Roberto Sottile, infine, da un'angolazione più ampia, la produzione consoliana è stata oggetto di analisi in una tesi di laurea discussa nel 2007/2008 con Rita Fresu ${ }^{\mathrm{I}}$.

Per procedere all'analisi è opportuno partire dall'intervento dell'accademico della Crusca Paolo D'Achille, in risposta alla domanda di una lettrice, apparso nel sito della Crusca il 29 gennaio 2019 nella sezione della Consulenza linguistica: La Cantantessa é una (e gli Studentessi sono solo canzonette)². D'Achille ripercorre la vicenda della parola in oggetto, cantantessa, e ne chiarisce lo statuto, offrendo così la spiegazione più completa sull'origine del suffissato che - come dimostra anche la domanda della lettrice - sortisce alcune perplessità, legate in qualche modo alle polemiche sui femminili di professione.

Il caso di cantantessa è peculiare ma offre la sponda per chiarire alcuni procedimenti linguistici sulla formazione dei femminili e sulla loro connotazione:

\begin{abstract}
In passato, è vero, c’è stata una tendenza a formare dei femminili in -essa (brigantessa, presidentessa), anche perché la norma tradizionale prescriveva l'uso del suffisso-essa per formare femminili da basi maschili in -e (principe/principessa, dottore/dottoressa), oltre che in -a (poeta/poetessa). Ma l'uso di questo suffisso, che è stato aggiunto, inutilmente sul piano morfologico, anche a nomi maschili in -o, spesso con una decisa connotazione spregiativa o ironica (medichessa, deputatessa, ecc.), è stato poi considerato discriminatorio nell'ottica del sessismo linguistico. Cosi, alle forme sopra citate si sono spesso affiancate quelle in cui la distinzione di genere è lasciata all'articolo e alle altre modalità di accordo grammaticale, come la presidente, che è da considerare ormai la forma standard 3 .
\end{abstract}

La parola è registrata nel dizionario dei Neologismi Quotidiani di Giovanni Adamo e Valeria Della Valle $(2003)^{4}$, nei Neologismi della Treccani (2008)5 e nell'Osservatorio neologico della lingua italiana (ONLI) ${ }^{6}$, con il significato di 'Cantante donna, cantautrice'; con il suffissato ci si riferisce in realtà esclusivamente alla cantautrice siciliana (per questo la Cantantessa è una nel titolo della consulenza; con studentessi, invece, ci si riferisce al titolo di un album del 2008 degli Elio e le storie tese), sebbene si possano reperire alcune occorrenze dell'appellativo riferite ad altre personalità: la maggior parte di esse reperibili in una stessa testata femminile online, con riferimento, per esempio, a Jennifer Lopez 7 ; altre presenti in testi narrativi ${ }^{8}$; una apparizione significativa, già segnalata da D'Achille, nel discorso della presidente della Società di Linguistica Italiana (SLI)9. 
L'origine dell'appellativo deriva invero da uno specifico episodio biografico riportato dalla stessa Consoli:

Fu un errore di un ingegnere del suono sudafricano che voleva dire di stare zitti perché la cantante doveva cantare, ma sembrandogli di rivolgersi a un uomo disse «la cantantessa». Mi piace perché non è un termine serio e non vale come dire «la cantante», quella che sa cantare. Io invece voglio passare come una che canta, una cantantessa appunto, che sta un gradino più sotto ${ }^{\mathrm{IO}}$.

Dal racconto dello stesso episodio che si leggeva nel sito ufficiale della Consoli apprendiamo che i presenti nello studio di registrazione stavano riflettendo - più o meno ironicamente - sul suffisso essa ${ }^{\mathrm{II}}$ :

Tutto successe nel periodo d'incisione, nello studio con l'ingegnere del suono sudafricano Allan Goldberg. Carmen con un suo amico cercava di far capire l'uso del suffisso 'essa' per il femminile. Così fecero un esempio per farglielo capire meglio, prendendo come cavia un cane di nome Mela e gli dissero che in italiano il Cane femmina si chiamava Canessa. Cosi Allan Goldberg sentendo che Cantante per Carmen suonava male, utilizzò l'appellativo Cantantessa.

I n Cantantessa l'accezione negativa del suffisso decade, perché si afferma come appellativo antonomastico pur mantenendo la sfumatura di modestia, più o meno retorica, con cui la Consoli vuole presentarsi e definirsi.

Permane un uso negativo del suffissato in alcuni contesti che non fanno specifico riferimento alla Consoli, come, per esempio, in questo brano:

Con una puntata di lancio più appetitosa di un banchetto nuziale, va in onda la nuova edizione dell'ormai caposaldo del sabato sera di casa Mediaset Amici di Maria De Filippi, con tutto il consueto codazzo di creature defilippiane: aspiranti cantanti e cantantesse, candidati ballerine e balleroni, insegnanti più o meno tassidermizzati, capisquadra ripescati nel dimenticatoio catodico, e, soprattutto, giudici, giudici e ancora giudici ${ }^{\mathrm{I} 2}$.

\section{Bésame Giuda}

Il testo, di cui la Consoli è autrice (lo è di tutte le proprie canzoni), è compreso nell'album Mediamente isterica: uscito il 29 ottobre 1998 per Cyclope/Polydor, è stato prodotto da Francesco Virlinzi, è ha ricevuto il riconoscimento del Disco d'oro, vendendo circa 80.000 copie; l'album è stato riedito dieci anni dopo, nel 2008 per la Universal, in versione deluxe (Mediamente isterica Deluxe Anniversary Edition), con gli stessi arrangiamenti ma ricantata da una Consoli più matura nella voce (cfr. Wikipedia). Il titolo dell'album viene proprio dal testo di Bésame Giuda, e vince su altre alternative possibili: Blu di metilene, Manuale delle piccole streghe, Misto incenso, Ingenuità dannata (che viene sempre dal testo di Bésame Giuda), Eclisse, Piccoli ritratti, Melodie del sottosuolo (con chiara allusione a Dostoevskij) ${ }^{\mathrm{I}}$.

Tra tutti questi, il titolo che alla fine è stato scelto appare il più significativo. L'album è notevole nella produzione della Consoli, sia per quanto riguarda la musica (per gli arrangiamenti, i campionamenti e i loop) sia perché è una galleria di racconti e anche di ritratti femminili: è qui che appare la figura, frutto della fantasia dell'artista, di Contessa Miseria, donna che lotta strenuamente contro la fine della giovinezza; o quella della donna del testo di Geisha, schiava di un uomo facoltoso; è un album che mette in musica vari dei cliché di cui ci ha parlato Francesca De Blasi nella sua introduzione. Il disco ha, inoltre, un forte valore iconico perché nella copertina Carmen Consoli è travestita da sirena 
surgelata e impacchettata come un pesce al supermercato (sul retro del disco è presente anche la data di confezionamento e quella di scadenza, il peso e le informazioni nutrizionali e di conservazione); all'interno del disco, la stessa Carmen - ma in versione umana - si aggira tra gli scaffali come a dire "posso anche comprarmi e divorarmi", secondo un progetto grafico che ha visto la collaborazione di Alberto Bettinetti, ideatore del progetto e con le fotografie di Remo di Gennaro ${ }^{\mathrm{I}}$.

Il singolo Bésame Giuda esce nel 1998 due settimane prima dell'album, in due edizioni che si distinguono per le tracce e per il materiale di confezionamento, ma entrambe mostrano in copertina Carmen Consoli che si fa baciare sulla guancia dal suo doppio (cfr. Discogs.com). Limmagine e il titolo anticipano il tema che è quello del tradimento - non del caso particolare dell'adulterio (come tiene a precisare la stessa Consoli) -, qui incarnato dal traditore per antonomasia, Giuda, e dall'episodio del bacio. Il celebre episodio è stato variamente rappresentato, e sono tanti gli esempi che si potrebbero fare guardando alla storia dell'arte, a partire da quel Bacio di Giuda affrescato tra il Izoz e il izo5 da Giotto nelle Storie di Cristo che si trovano nella Cappella degli Scrovegni a Padova.

Il verbo spagnolo del titolo bésame (ma si trova anche senza accento) se da una parte cita a distanza la canzone del 1940 Bésame mucho di Consuelo Velásquez (con la quale nei concerti dal vivo Carmen sfuma e attenua il testo e la musica della sua canzone), dall'altra modifica e gioca con l'espressione cristallizzata bacio di Giuda con la quale si indica l'episodio della Passione di Cristo.

Il Giuda consoliano è all'inizio del testo un Giuda tradizionale, che è possibile accostare a quello biblico e anche dantesco: la punizione che Dante riserva a Giuda nel trentaquattresimo canto dell'Inferno (vv. 6r-63) è infatti la più atroce, maciullato com'è a testa in giù da Lucifero, una sorte simile a quella che la voce narrante della canzone avrebbe probabilmente voluto per il proprio Giuda' ${ }^{\mathrm{I}}$ :

"Quell'anima là su c'ha maggior pena",

disse'l maestro, "è Giuda Scarïotto,

che 'l capo ha dentro e fuor le gambe mena"'

Evocate le suggestioni del tema, vediamo il testo della canzone. La donna elenca le nefandezze del proprio traditore in un processo di presa di coscienza con una autonarrazione che la mostra - se non risorta come Cristo - almeno sopravvissuta al punto di potersene vantare:

\author{
Sopravvissuta a quest'ultima prova \\ in fondo posso vantarmi di essere una donna con la D maiuscola \\ certamente ho fatto tesoro \\ dei tuoi insegnamenti \\ e posso ben dire \\ di essere una donna con la D maiuscola \\ di essere una donna con le carte in regola
}

La riappropriazione di sé stessa come donna passa dalla capitalizzazione dell'iniziale di Donna, che ha valore accrescitivo (moralmente) ed enfatico; lo stesso di formule come "la Storia con la S maiuscola" e via dicendo. Il verso è ripetuto e rafforzato da una variante che esprime lo stesso concetto "con le carte in regola", locuzione che passa dal lessico burocratico al linguaggio corrente (carta 'documento').

Il tono del testo assume note ironiche e dipinge una donna che cerca di autoconvincersi ma che poi cede al rimprovero di sé stessa: "D come dannata ingenua" ripete nel refrain, e lì la stessa $D$ di Donna individua, sfruttando l'espediente dell'alfabeto telefonico (in altre parole lo stesso della $D$ di 
Domodossola, esempio diventato famoso grazie a un noto programma condotto da Mike Bongiorno), è una donna ingannata e vittima di "raggiro". Anche dannata contiene entrambe le accezioni possibili, sia di 'condannata' ma anche di semplice rafforzativo, svuotato semanticamente, per accompagnare il sostantivo ingenua 'privo di malizia'.

Le mistificazioni del Giuda in questione (chiunque esso sia) sono elencate sfruttando verbi e sostantivi dal forte impatto semantico e fonosimbolico, come tramare 'tessere inganni', strisciare 'comportarsi in modo degradante e servile' e raggiro 'inganno costruito a scapito della buona fede altrui', di cui si nota la presenza reiterata della vibrante anche nei gruppi consonantici tr-e str-.

Nella seconda parte del testo si esplicita la dannazione a cui è costretta la donna che chiede al suo Giuda di baciarla ancora, come se non potesse fare a meno dell'inganno, o come se qui il Giuda biblico e dantesco cedesse il passo a quello del testo apocrifo del Vangelo di Giuda, secondo il quale l'Iscariota, da tredicesimo apostolo, non tradisce ma piuttosto asseconda la volontà di Cristo in modo da permetterne la morte, e quindi la resurrezione (prospettiva ampiamente usufruita in letteratura e musica; porto due esempi a me noti: il controverso romanzo di Nikos Kazantzakis, Lultima tentazione, I960 e la canzone di Bob Dylan, With God on our side, nell'album The Tymes They Are a-Changin', I964, Columbia records).

Quando la donna si presenta consapevole, di sé stessa e dell'inganno perpetrato a sue spese si definisce "una Donna con la D maiuscola" e "mediamente isterica".

Ma lasciamo la parola all'autrice che cosi commenta il proprio testo:

È una canzone sul tradimento, un grande tradimento: uno di quelli che ti segnano per moltissimi anni e forse per tutta la vita. Da un lato lascia emergere l'ironia, vedi "Giuda baciami ancora" o la trasformazione finale in Bésame mucho, e dall'altro è una specie di autocritica accusatoria per essere stata tanto ingenua da incappare in una così enorme frode. Oggi come oggi non ha poi importanza chi fosse il Giuda a cui mi riferivo o se il tradimento fosse avvenuto proprio nei termini in cui, sdegnata, lo vedevo: conta, invece, che il pezzo esprima alla perfezione lo stato d'animo di una persona raggirata, che prova rabbia ma che comunque fa tesoro dell'esperienza negativa. E che quindi cresce ("Sopravvissuta a quest'ultima prova / In fondo posso vantarmi / Di essere una donna con la D maiuscola") pur diventando più cinica. Il Per quanto tempo hai strisciato tra le mie lenzuola" non va interpretato alla lettera, il sesso non c'entra nulla: per me le lenzuola simboleggiano l'intimità in senso lato, al punto che mi capita di portarmene di mie anche in hotel. L'ho scelta come prima traccia perché l'inganno, assieme alla conseguente disillusione, è il tema che domina l'intero album ${ }^{\mathrm{I} 7}$.

\section{Mediamente isterica}

Soffermiamoci a questo punto sull'aggettivo isterico, che non a caso è presente in sintagma con l'avverbio mediamente in funzione di attenuazione.

Tale attenuazione appare necessaria alla Cantantessa che, riflettendo sull'isteria, la intende non solo come condizione femminile, per quanto parta dal dato fisiologico della crisi premestruale, ma più ampio, attribuendo un senso generale di subbuglio, come si legge nel suo sito:

Mediamente isterica è un altro stato d'animo, ma non contraddittorio come per Confusa e felice. Lidea è venuta dalla settimana che precede il ciclo, in cui lo scompenso ormonale agisce sul sistema nervoso di noi donne e provoca una fase depressiva durante la quale non siamo molto padrone delle nostre reazioni. 
L'aggettivo è registrato nei Vocabolari della Crusca a partire dall'impressione del i6gi, cioè la Terza $\mathrm{Crusca}^{\mathrm{I}}{ }^{8}$, consultabile, insieme alle altre impressioni, anche in modalità sinottica, al sito della Lessicografia della Crusca in rete. Il lemma presenta una definizione neutra, ed è esemplificato da un testo tecnico di argomento, per cosi dire, medico $^{19}$.

La storia dell'isteria ha una ricaduta evidente sulle accezioni negative con cui ancora si usano il sostantivo o l'aggettivo perlopiù riferiti alla donna. Riassumendo, dopo Ippocrate la natura dellisteria

cominciò a essere studiata con indirizzo scientifico solo nel $17^{\circ}$ sec., quando C. Lepois per primo la mise in rapporto con il sistema nervoso e T. Sydenham sostenne l'importanza dell'emozione nella genesi delle sue manifestazioni e ne escluse la genesi uterina, notando che l'i. può svilupparsi anche nel sesso maschile" (Treccani, Enciclopedia, s.v. isteria)

Alla stessa conclusione, che esclude l'ipotesi uterina, giungono successivamente anche le scuole di psichiatria a indirizzo medico.

Torniamo ai vocabolari: per una ricostruzione storico-linguistica, si mostra sempre utile la consultazione del Dizionario della Lingua Italiana di Nicolò Tommaseo e Bernardo Bellini uscito nel I86I e disponibile dal 2015 anche in versione online. Questo vocabolario ha come caratteristica più peculiare e notoria quella di restituirci i giudizi del suo autore principale, Niccolò Tommaseo. Proprio per questa caratteristica, e visto una generale misoginia che trapela più o meno consapevolmente da altre definizioni, oltre naturalmente alla percezione dell'epoca, non stupirà che alla definizione di isteria si legge che si tratta di una malattia "a cui vanno sovente soggette le donne".

Guardando al corpus esaustivo dei testi della Consoli si ricava che l'aggettivo è utilizzato altre tre volte nel brano La dolce attesa dell'album Eva contro Eva del 2006, altro lavoro in cui, a partire dal titolo, emergono varie figure femminili. Nel brano in questione si parla di una gravidanza isterica, indotta nella protagonista da pressioni sociali esterne, da chi ritiene che la donna possa realizzarsi soltanto nell'esperienza della maternità.

Al terzo mese di gravidanza isterica
Già sul viso i morbidi tratti di maternità
Diceva Maddalena sarebbe un nome particolare insieme a Sofia
Nel caso fosse maschio Vincenzo Maria
Al sesto mese di gravidanza isterica
Tutti ritennero fosse opportuno non scomodare la verità
Insidiati dai rimorsi per averle dato il tormento
Finché desse alla luce una creatura entro l'etá feconda

Mentre aspettava il lieto evento

Che mai avrebbe avuto luogo

Comprava abiti premaman e una culla di legno

Come quelle di una volta

Le si leggeva in faccia smisurata felicità

Per la dolce attesa

Al nono mese di gravidanza isterica

Tutti mantennero la messa in scena invariata per viltà

Sarebbe stata questione di giorni ed avrebbe chiarito da se

L'increscioso equivoco di cui era la sola ed unica artefice 
Il significato di isterico nell'espressione mediamente isterica di Bésame Giuda è più neutro e più vicino al senso comune registrato nei vocabolari moderni dell'italiano contemporaneo. Cosi per esempio nel Treccani e nel Grande Dizionario dellitaliano di Tullio De Mauro (GRADIT), disponibile anche in una versione online sul sito della rivista "Internazionale" come Nuovo De Mauro. Il GRADIT, in particolare, e come caratteristica distintiva, offre una classificazione fatta su base statistica che organizza i significati in base al loro àmbito d'uso, che nel caso di isterico sono quello tecnico (TS = testi specialistici) e quello comune $(\mathrm{CO}=$ comune $)$.

Per concludere. La donna consoliana mediamente isterica emerge come una figura multiforme, o almeno duplice, debole e volubile, ma anche forte e consapevole al punto di smascherare il suo carnefice e la sua condizione pur rischiando, allo stesso tempo, di rimanere impantanata in essa. Sicuramente l'aggettivo isterica non va letto con valore negativo anche in forza dell'avverbio e della sua funzione attenuativa. La donna mediamente isterica è una donna ancora confusa (come lo è quella di Confusa e felice), in subbuglio ormonale, ma con moderazione, mediamente, in una condizione condivisa e indipendente dal sesso di chi la vive. Quello femminile è "un punto di vista" dice la Consoli:

Chi pensa che dai miei testi emerga un odio, una collera immensa nei confronti degli uomini, sbaglia. Parto spesso da un punto di vista femminile perché mi viene più facile descrivere determinati dettagli, mi sembra di essere più credibile. Contessa miseria è Dorian Gray, ma a Oscar Wilde veniva probabilmente più spontaneo rapportarsi con la vanità maschile

Bésame giuda sembra veicolare un messaggio che dalla condizione di una donna - per quanto "con la D maiuscola" - possa valere per le donne (tutte maiuscole) e per tutti. Femminile si - dunque - ma anche plurale e universale.

Note:

I. Si vedano almeno Giuseppe Antonelli, Ma cosa vuoi che sia una canzone. Mezzo secolo di italiano cantato, Bologna, il Mulino, zoro; Roberto Sottile, Dialetto e canzone. Uno sguardo sulla Sicilia di oggi, Firenze, Cesati, 20r8; Silvia Piras, "Vorrei dire due parole". Analisi lessicale e stilistica della lingua delle canzoni di Carmen Consoli, Tesi di Laurea, Università di Cagliari, Rel. Rita Fresu, a.a. 2007/2008 (ringrazio Silvia Piras e Rita Fresu per avermi agevolato la lettura della tesi inedita). Per i principali riferimenti bibliografici sulla lingua della canzone rimando agli studi già evocati nei contributi di Chiara Murru e Francesca Cialdini in questo stesso numero. Ringrazio Francesca Tiselli per il proficuo scambio di idee (e non solo) sulla Cantantessa.

2. "C.A., da Cagliari, ci chiede «se è lecito utilizzare la parola cantantessa, [...] ritenendo che la parola cantante sia sostantivo maschile e femminile»"; cfr. Paolo D'Achille, La Cantantessa é una (e gli studentessi sono solo canzonette), ora anche in "Italiano digitale", VII, 2019/I (gennaio-marzo), pp. I3-I5.

3. Ivi, p. I3.

4. Giovanni Adamo e Valeria Della Valle, Neologismi quotidiani. Un dizionario a cavallo del millennio 1998-2003 Firenze, Olschki, 2003, s.v., p. 222 con esemplificazione: "Con Per niente stanca, una rabbiosa canzone sull'Aids, si è aperto il concerto della cantautrice («cantantessa», come si definisce lei ironicamente) catanese [Carmen Consoli] che si è esibita di fronte a una platea entusiasta, in larga maggioranza diciottenni e ventenni" ("Corriere della sera", I3 marzo I999, p. 52, Spettacoli). 
5. Il Vocabolario Treccani. Neologismi. Parole nuove dai giornali, Roma, Istituto Enciclopedico Treccani, 2008, secondo i quali la prima apparizione si trova in Gloria Pozzi, "Noi giovani siciliani con il rock contro la mafia", "Corriere della sera", 23 maggio I997, p. 35, Spettacoli.

6. Banca dati dell'Istituto per il lessico intellettuale europeo e storia delle idee (Iliesi) del CNR ONLI, Osservatorio neologico della lingua italiana.

7. Si tratta della rivista marieclaire.com, per esempio nell'articolo Il tubino di Jennifer Lopez fa evaporare le cifre sulla carta d'identità del in novembre 2019 si legge: "Imprenditrice, cantantessa, attrice, JLo ha poi raccontato la news forse più curiosa sulla sua partecipazione al film Hustlers" e così in altri articoli, in riferimento, per esempio, a Céline Dion. L’uso appare dunque circoscritto, imputabile allo stile dei redattori della rivista, e estensivo rispetto all'uso antonomastico riferito a Carmen Consoli.

8. Da GoogleBooks ricavo le seguenti attestazioni: I) Matteo Carletti, Storie vere inventate. Goliardici racconti, 20I2: "C'era una Volta, nell'Africa più misteriosa, una formichina che covava un grande sogno. Divenire una cantantessa"; 2) Stella Pulpo, Fai uno squillo quando arrivi, 2017: "Apprendo cosi che questa sera c'è un concerto di una cantantessa reggae di cui ignoro l'esistenza e che vanno TUTTI".

9. "[...] vorrei essere chiamata «la presidente», come da anni suggeriscono le Raccomandazioni per un uso non sessista della lingua italiana. Sono ben consapevole che gli usi linguistici privati e informali non si impongono per legge, ma mi fa piacere rispondere a chi mi chiede la mia preferenza. Chi trova strana la formula «la presidente» si chieda: Mina è «un cantante»o «una cantantessa»? Molti auguri di buon lavoro e di buon anno dalla vostra nuova presidente". (Anna M. Thornton, Presidente della SLI, nel Bollettino, XXXIV, 20I5, 2; cito da D'Achille, La Cantantessa, cit., p. I4).

Io. Gianni Bonina, Carmen Consoli, ritorno in Sicilia: "È tempo di dire grazie alla mia terra", in palermo.repubblica.it, 9 marzo 2015 .

II. Il brano è citato da D’Achille, La Cantantessa, cit., p. iz (non è più reperibile nel sito ufficiale della Consoli, www.carmenconsoli.it), a cui rimando anche per i valori del suffisso -essa riassunti dallo studioso nel suo contributo.

I2. Cito dalla banca dati ONLI, Osservatorio neologico della lingua italiana, cit. (l'articolo è apparso nel "Sole 24 Ore", Io aprile 20I6, p. 42, Domenica).

I3. Si veda Elena Raugei, Carmen Consoli, Fedele a se stessa, Roma, Arcana, 20 oro.

I4. Ibidem.

15. Non sono rari i riferimenti letterari nella produzione della Consoli, dal mito classico alla letteratura più moderna; due esempi per i due estremi: da una parte Orfeo e Euridice nel testo di Orfeo (2000), mito rivisitato con lieto fine; dall'altra La lunga vita di Marianna Ucria di Dacia Maraini che influenza la composizione di Sulla mia pelle (I996, su cui cfr. anche Dario Accolla, Mito e regionalitá nell'onomastica di Carmen Consoli, in "Il nome nel testo", XIV, 20I2, pp. I4I-I50: I44). Per quanto riguarda il caso specifico di Dante, il poema dantesco è spesso evocato dalla Cantantessa nelle sue interviste; tra tutte mi pare particolarmente significativa questa dichiarazione: "A scuola quando dovevo imparare a memoria la «Divina Commedia», la trasformavo in una canzoncina: «Nel mezzo del cammin di nostra vita, mi ritrovai per una selva oscura...» (recita i versi canticchiando, ndr)" in Luca Castelli, La Carmen Consoli rigenerata: "Il mio amore non è piu di plastica", in "Corriere Torino", I luglio 20I9. 
I6. Il testo è quello di Petrocchi (a parte il su che l'editore accenta nella sua edizione): Dante Alighieri, La Commedia secondo l'antica vulgata, a cura di Giorgio Petrocchi, Firenze, Le Lettere, I994, voll. 2-4 (prima ediz.: Milano, Mondadori, I966-1967), p. 590. Gli altri due traditori, Bruto e Cassio, hanno "il capo di sotto" (Inf. XXXIV, v. 64). Giuda è l'apostolo traditore come narra il Vangelo ( Matth. 26, I4-6): "Egli sta nella bocca centrale di Lucifero, come il peggiore non solo dei traditori, ma di tutti i dannati dell'inferno. Di lui tuttavia Dante dice solo il nome, senza alcun commento. Quasi che a tale colpa e a tale nome nulla fosse possibile aggiungere" (cito dal commento di Anna Maria Chiavacci Leonardi, Dante Alighieri, La Divina Commedia, Inferno, Milano, Mondadori, I99I, nota 62, vv. 6I-63, p. IoI8).

I7. Federico Guglielmi, Quello che sento. Il mondo, i pensieri, la musica di Carmen Consoli, Firenze, Giunti, 200I (seconda ed. 2006), p. I50.

I8. Consultabile, insieme alle altre impressioni, anche in modalità sinottica, al sito della Lessicografia della Crusca in rete.

I9. Si tratta del cosiddetto Libro della cura delle malattie attribuito al volgarizzatore Zucchero Bencivenni, che è probabilmente una fonte fantasma, frutto delle falsificazioni di Francesco Redi, su cui si veda Guglielmo Volpi, Le falsificazioni di Francesco Redi nel "Vocabolario della Crusca", in "Atti della R. Accademia della Crusca per la lingua d'Italia”, a.a. I9I5-I9ı6, in particolare pp. 73-76, Antjie Bielfeld, Methoden der Belegsammlung für das "Vocabolario della Crusca", Tübingen, Max Niemeyer, I996, a p. I85, ma anche Rossella Mosti, I falsi del Redi visti dal cantiere del "Tesoro della Lingua italiana delle Origini", in "Bollettino dell'Opera del Vocabolario Italiano", XIII, 2008, pp. 38I-97 e altra bibliografia ivi citata.

\section{Cita come:}

Veronica Ricotta, "Una donna con la D maiuscola". Analisi di Bésame Giuda di Carmen Consoli, "Italiano digitale", 2019, Xl, 2019/4 (ottobre-dicembre)

DOI: $10.35948 / 2532-9006 / 2020.3163$

Copyright 2019 Accademia della Crusca

Pubblicato con licenza creative commons CC BY-NC-ND 